\section{Mehr Aufmerksamkeit für chronische Schmerzen}

\author{
In der speziellen Schmerzmedizin herrscht auch im Jahre 2016 in Deutschland \\ noch immer Notstand. Das belegen die Zahlen des Barmer GEK Arztreports. \\ Zur Stärkung der Schmerzmedizin und der patientenindividuellen Behand- \\ lung sollten chronischen Schmerzen mehr Aufmerksamkeit geschenkt und \\ sie als Krankheit anerkannt werden.
}

$\mathrm{N}$ ach aktuellen Daten leiden 23 Millionen Deutsche $(28 \%)$ an chronischen, meist nicht tumorbedingten Schmerzen. Alte Menschen sind sogar zu über $50 \%$ betroffen. Dies bestätigt auch der neueste Arztreport der Barmer GEK von 2016, nach dem bei über 30 Millionen Deutschen Schmerzdiagnosen gestellt werden. Etwa 2-3 Millionen Patienten benötigen eigentlich eine spezialisierte Schmerztherapie nach definierten Qualitätsstandards, doch stehen hierfür derzeit nur knapp 1.000 niedergelassene Schmerzmediziner zur Verfügung.

\section{Nachwuchsprobleme in der Schmerzmedizin}

Für den Ludwigshafener Schmerztherapeuten Dr. Oliver Emrich aus Ludwigshafen belegen die genannten Zahlen: "Schmerzmedizinische Versorgung ist weiterhin in Deutschland nicht sichergestellt." Hinzu komme, dass die Hauptlast der speziellen Versorgung niedergelassene Ärzte tragen - über 95\% dieser Patienten müssten in diesem Sektor versorgt werden. Gesundheitspolitik und KVen könnten den Sicherstellungsauftrag nicht wahrnehmen, da das Fehlen eines Facharztes für Schmerzmedizin dies verhindere. "Jeder, der Schmerzmedizin in seiner Praxis betreibt, ist ganz auf sich alleine gestellt." Auch die Qualifikation in Ausbildung und Versorgung sind immer noch rudimentär, da erst seit 2016 an Universitäten Schmerzmedizin als Ausbildungspunkt berücksichtigt werde. Gleichermaßen desaströs sieht Emrich das Fehlen wirtschaftlicher Strukturen für eine zukunftsorientierte schmerzmedizinische Versorgung. In vielen KV-Bezirken erlebe man weitere Reglementierungen und heruntergesetzte Budgets. Im Verteilungskampf um begrenzte Mittel zögen Schmerzmediziner ohne eine ausreichende Lobby den Kürzeren. Als Meilensteine der Schmerztherapie in Deutschland nannte Emrich die erste bundesweite Schmerztherapievereinbarung aus dem Jahre 1994 und die Aufnahme der Schmerztherapie in den EBM in 2005.

\section{Qualitätsanforderungen erfüllt}

Mit heute über 129 regionalen Schmerzzentren sieht sich die aus dem 1994 gegründeten Schmerztherapeutischen Kolloquium (StK) entstandene Deutsche Gesellschaft für Schmerzmedizin (DGS) als ,VersorgerGesellschaft im Auftrag der Patientenversorgung “. Mit der jährlichen Rezertifizierung der DGS-Mitglieder zum Algesiologen sowie regelmäßigen Fortbildungen, interdisziplinären Schmerzkonferenzen und einer qualitativ hochwertigen Vernetzungsstruktur „werden die Qualitätsanforderungen von KBV und BÄK eigentlich noch übertroffen", so Emrich. Allerdings ergebe sich aus dem - nach einem bio-psychosozialen Krankheitsverständnis von Schmerzen - erforderlichen komplexen Standard für Diagnostik und Therapie für die immense Zahl der Schmerzpatienten aus Sicht der DGS eine wesentliche Forderung: „Wir können die Schmerzmedizin, so wie wir sie brauchen, nur etablieren, wenn wir den Facharzt für Schmerzmedizin schaffen."

Unterstützung bekam Emrich für diese Forderung von Professor Joachim Nadstawek, Vorstandsmitglied des Berufsverbandes der Ärzte und Psychologischen Psychotherapeuten in der Schmerzund Palliativmedizin in Deutschland (BVSD): „Nur durch die Einführung eines Facharztes ist die Ermittlung der Bedarfsplanung möglich." Er sieht in der völlig ungenügenden Zahl und gleichzeitiger Überalterung der Schmerzmediziner ein massives Nachwuchsproblem.

„Eine Crux ist nach wie vor, dass die Schmerzmedizin nicht in der Bedarfsplanung auftaucht", sagte Nadstawek. Als beispielhaft sieht er etwa die Vereinbarung des BVSD mit Kassen und regi- onaler KV in Schleswig-Holstein, künftig Kassensitze mit schmerztherapeutischer Kompetenz nur durch Kollegen mit schmerztherapeutischer Kompetenz zu besetzen.

\section{Patientenwohl im Blick}

Auf die dramatische Zunahme der Zahl chronischer Schmerzpatienten in Deutschland in der letzten Dekade verwies auch Privatdozent Dr. Michael Überall aus Nürnberg, Vizepräsident der DGS und Präsident der Deutschen Schmerzliga. Lag deren Anteil an der Gesamtbevölkerung im Jahre 2006 noch bei $17 \%$, kam eine Untersuchung aus 2009 bereits auf $23 \%$, eine weitere aus 2014 auf $29 \%$ und der aktuelle Barmer GEK-Report (2016) bereits auf $33 \%$, also einem Drittel der Gesamtbevölkerung. Eine Berechnung der direkten und indirekten Kosten alleine durch Kreuzschmerzen ergab schon im Jahre 2009 für Deutschland die astronomische Summe von knapp 49 Milliarden Euro, erläuterte Überall. Er sieht als Ursache dieser Entwicklung vor allem das aus dem Blickfeld geratene Patientenwohl. Nach einer Forsa-Umfrage aus dem Jahr 2011

_ist die Mehrheit der Schmerzpatienten (59\%) der Ansicht, dass es in der Öffentlichkeit an Verständnis für ihre Situation mangelt,

_ sind sich $48 \%$ der Ärzte über ihre Strategie unsicher, wenn Schmerzen trotz Behandlung länger persistieren,

_erhalten $38 \%$ aller Schmerzpatienten trotz jahrelanger Behandlungsversuche nach eigener Einschätzung nicht die richtige Therapie und

- werden nur $8 \%$ aller chronischen Schmerzpatienten von einem qualifizierten Schmerztherapeuten behandelt.

Überalls Forderungen: Chronische Schmerzen erfordern mehr Aufmerksamkeit, sie müssen als Krankheit anerkannt werden und verlangen von Anfang an eine gezielte Behandlung. Im Mittelpunkt müsse der individuelle Behandlungserfolg des chronisch kranken Menschen stehen.

Dr. Andreas Häckel

Symposium „Schmerzmedizin in Deutschland wo kommen wir her, wo wollen wir hin?"

Frankfurt am Main, 3.3.2016 